\section{Rheumatological diseases and cancer: the hidden variable of radiation exposure}

\author{
Eugenio Picano, ${ }^{1}$ Richard Semelka, ${ }^{2}$ James Ravenel, ${ }^{3}$ \\ Marco Matucci-Cerinic ${ }^{4}$
}

Recently, several different meta-analyses have shown that rheumatological diseases —including systemic sclerosis (SSc), systemic lupus erythematosus, rheumatoid arthritis and psoriatic arthritis-are associated with an increased risk of cancer, in particular non-Hodgkin's lymphoma, lung and liver cancer. ${ }^{1-6}$ Several hypotheses have been advanced to explain this finding, including immune dysregulation, biological therapy and exposure to common environmental risk factors. ${ }^{7}$ These data may suggest the inclusion of medical radiation as a potential, and verifiable, iatrogenic risk linking SSc and cancer. This malignant induction may be potentiated by the concomitant use of drugs, such as methotrexate and cyclophosphamide, or drugs such as paracetamol. In fact, these drugs are known to amplify the genotoxic effects of medical radiation. In addition, in some rheumatic diseases, the exposure to medical radiation is associated with an intrinsic higher vulnerability for DNA instability.

Frauenfelder et $a l^{8}$ present the prospective validation of a dedicated, 9-slice high resolution computed tomography (HRCT) protocol with reduced radiation dose for the detection of interstitial lung disease in SSc patients. This paper addresses clearly the fact that the dose reduction is becoming today a hot issue in clinics. Herein, we wish to draw the attention of the rheumatological community to the hypothesis that, in rheumatological patients, the use of medical radiation may pose an additional risk for cancer development, possibly potentiated by the concurrent use of antirheumatic drugs.

\footnotetext{
${ }^{1}$ Institute of Clinical Physiology, CNR, Pisa, Italy; ${ }^{2}$ Department of Radiology, University of North Carolina, Chapel Hill, North Carolina, USA; ${ }^{3}$ Department of Radiology and Radiologic Sciences, Medical University of South Carolina, Charleston, South Carolina, USA;

${ }^{4}$ Division of Rheumatology AOUC, Department of Experimental and Clinical Medicine, University of Florence, Florence, Italy

Correspondence to Dr Marco Matucci-Cerinic, Division of Rheumatology AOUC, Department of Experimental and Clinical Medicine, University of Florence, Florence 50139, Italy;

marco.matuccicerinic@unifi.it
}

\section{MEDICAL RADIATION AS A} POTENTIAL CARCINOGEN

Medical radiation is suspected to contribute to the development of cancer. According to the linear no-threshold theory, all radiation poses some risk of cancer induction, although the magnitude of this risk remains unclear, particularly at very low doses. The average background exposure of a US citizen approximates 3.0 milliSievert $(\mathrm{mSv}$, corresponding to the radiological dose equivalence of 150 chest $\mathrm{X}$-rays) per person per year, as estimated in 2006.9 Doses of examinations commonly employed in rheumatology patients range from 2 to $8 \mathrm{mSv}$ for a chest CT (100-400 chest X-rays) to $10-40 \mathrm{mSv}$ for a myocardial perfusion scintigraphy (500-4000 chest $\mathrm{X}$-rays). ${ }^{10}$ The radiological risk is considered cumulative in nature; thus, each exam using radiation increases the risk. Furthermore, children are more sensitive to radiation than adults, and females are more sensitive to radiation risks than males. ${ }^{11}$ Rheumatological patients, often young at disease onset and very frequently female, are therefore more prone to radiation related risks. The chronic nature of most rheumatological diseases also renders our patients more vulnerable to experiencing the cumulative effects of multiple and repeated X-ray studies. Although imaging is a fundamental tool in the diagnosis and monitoring of disease evolution and response to therapy, ${ }^{12}$ we wish to draw attention to the need of appropriate riskbenefit analysis for the type and frequency of studies.

The available epidemiological evidence linking radiation exposure to increased cancer risk is well established for doses $>10-50 \mathrm{mSv}$, which may be experienced by our patients during one admission, after one episode of care, or in some circumstances a single examination. ${ }^{12}$ A recent study of 180000 people exposed to CT scan in the UK found an increasing risk of leukaemia and brain cancer with increasing radiation exposure, with cumulative ionising radiation doses from three head CTs tripling the risk of brain tumours and 5-10 head CTs tripling the risk of leukaemia. ${ }^{13}$ Among 680000 Australians exposed to a
CT scan when aged 0-19 years, cancer incidence was increased by $24 \%$ compared with the incidence in over 10 million unexposed people. ${ }^{14}$ Epidemiological evidence is also emerging showing a link between increased radiation exposure and subsequent increased risk of cancer in adult populations of patients with ischaemic heart disease. ${ }^{15}$

In rheumatic patients, imaging of the chest (an anatomic region that includes the radiosensitve organs lung, female breast and bone marrow) is commonly performed. ${ }^{16}$ The lungs may experience relatively high organ doses from right heart catheterisation for suspected pulmonary arterial hypertension, ${ }^{17}$ and from chest CT employed for detection of lung involvement. ${ }^{18}$ Gallium scintigraphy previously often used to assess pulmonary disease activity, ${ }^{19}$ and cardiac stress scintigraphy with thallium or technetium-based tracers such as sestamibi to evaluate coronary and myocardial involvement. ${ }^{20}$ The organ sites where imaging has been performed often correspond to where malignancy has developed. Cumulative radiation contributes to the increase in cancer. ${ }^{21}$ Risk estimates are further complicated, and therefore more subject to being contested because cancer induction often occurs years or decades after exposure.

\section{VULNERABILITY OF}

\section{RHEUMATOLOGICAL PATIENTS TO} DAMAGING EFFECTS OF RADIATION: \section{AGE, GENDER, GENES AND DRUGS}

The dose-risk curve in radiation risk is established from large epidemiological data banks, including $>100000$ survivors of the atomic bomb, $>400000$ nuclear power plant workers and >800000 patients exposed to diagnostic medical radiation. The challenge ahead is to translate the generic population risk obtained from epidemiological data into a personalised risk. Several genetic and pharmacological variables can affect the variability of damage observed for any given level of radiation. For instance, radiation-induced chromosomal damage is amplified by genetic polymorphisms or gene mutations (such as BRCA1 and BRCA2) of genes involved in DNA repair. ${ }^{22}$ A similar situation may occur in rheumatological patients in whom epidemiological, genetic and pharmacological factors may enhance vulnerability to a given dose of radiation, making the linear relationship linking risk to dose progressively steeper (figure 1). First, rheumatological patients are much more often women (with a 4:1 ratio), who are $38 \%$ more vulnerable to radiation than men. ${ }^{11}$ Second, the age at first 


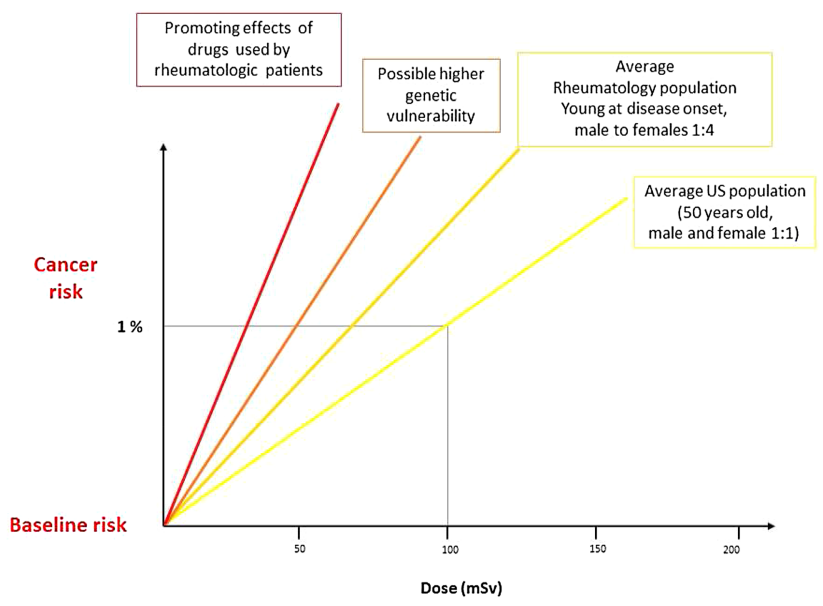

Figure 1 The population risk estimated for an average population is in reality derived from a spectrum of risks, for instance with higher risks being associated with female gender, younger-than-average age, mutation of genes involved in DNA repair and presence of other environmental carcinogens such as alkylating agents or some anti-inflammatory drugs. The 'average' risk of one extra-cancer for every $100 \mathrm{mSv}$ radiation exposure can in this way increase significantly, even detectable with a classic epidemiology approach on a few thousand patients.

diagnosis is on average relatively young, and radiation vulnerability increases with younger age, being twice as high at 30 years than at 50 years of age. ${ }^{15}$ Third, data suggest a particular sensitivity of DNA from rheumatology patients, with defective repair ability. Ex vivo studies in peripheral blood lymphocytes from children with systemic lupus, juvenile rheumatoid arthritis and SSc show a twofold increase in biomarkers of DNA damage as well as delayed repair of DNA damage after irradiation, suggesting an intrinsic increased vulnerability to effects of ionising radiation. ${ }^{23}$ The increased vulnerability to radiation has also been observed for non-cancer effects evoked by high-dose therapeutic radiation exposures, such as fibrosis after radiotherapy, which may precipitate SSc or cause a generalised worsening of the underlying disease, promoting oxidative stress, hypoxia and microvascular damage. ${ }^{24}{ }^{25}$ Fourth, rheumatological patients frequently use alkylating agents such as methotrexate and cyclophosphamide that are known biological modifiers of radiation-induced DNA damage, ${ }^{26}$ which may potentiate supra-additive genotoxic effects with radiation. $^{27} 28$ In addition, some antiinflammatory drugs, such as paracetamol, have inhibitory effects on DNA repair in mammalian cells and may contribute to genotoxicity in humans. ${ }^{28} 29$

\section{TAKE-HOME MESSAGES FOR THE RESEARCHER AND THE CLINICIAN}

The radiation to cancer-induction relationship can be prospectively tested with further data mining, including reference doses (when actual delivered doses are not available), genotyping, and characterisation of type and use of concomitant medications, as variables. In practical terms, the issue of radiation risk should stimulate re-evaluation of imaging strategies, moving away from standardised one-sizefits-all screening for disease to symptombased, patient-centric or tailored strategies emphasising risk-benefit analysis on an individual basis, including justification and optimisation of imaging protocols. In addition, the diagnostic strategy in patients, with SSc for example, might substantially shift towards radiation-sparing approaches whenever possible, taking full advantage of the technological advances of the last 5 years. In SSc, recent studies have shown that screening for interstitial lung disease with a limited number of high resolution CT slices may be sufficient for screening and follow-up. ${ }^{8} 30$

Usually, for the diagnosis of pulmonary hypertension, right heart cathetherisation is mandatory but in practice transthoracic
Figure 2 The diagnostic potential of four commonly faced challenges in rheumatology patients: lung fibrosis, pulmonary hypertension, myocardial ischaemia and bone osteoporosis. For each, the imaging menu includes an ionising (left) and non-ionising (right) option, with inherent differences in complexity, cost and availability. With the ionising approach, the true radiation exposure can vary up to 10 -fold compared with the reference dose depending on the technology and technique used: the dose administered should always be known and recorded in patients' records. Reference doses are taken from. ${ }^{9} 38$ Ultrasound osteoporosis image by courtesy of Echo-light and Casciaro S.

\section{Rheumatologic patients}

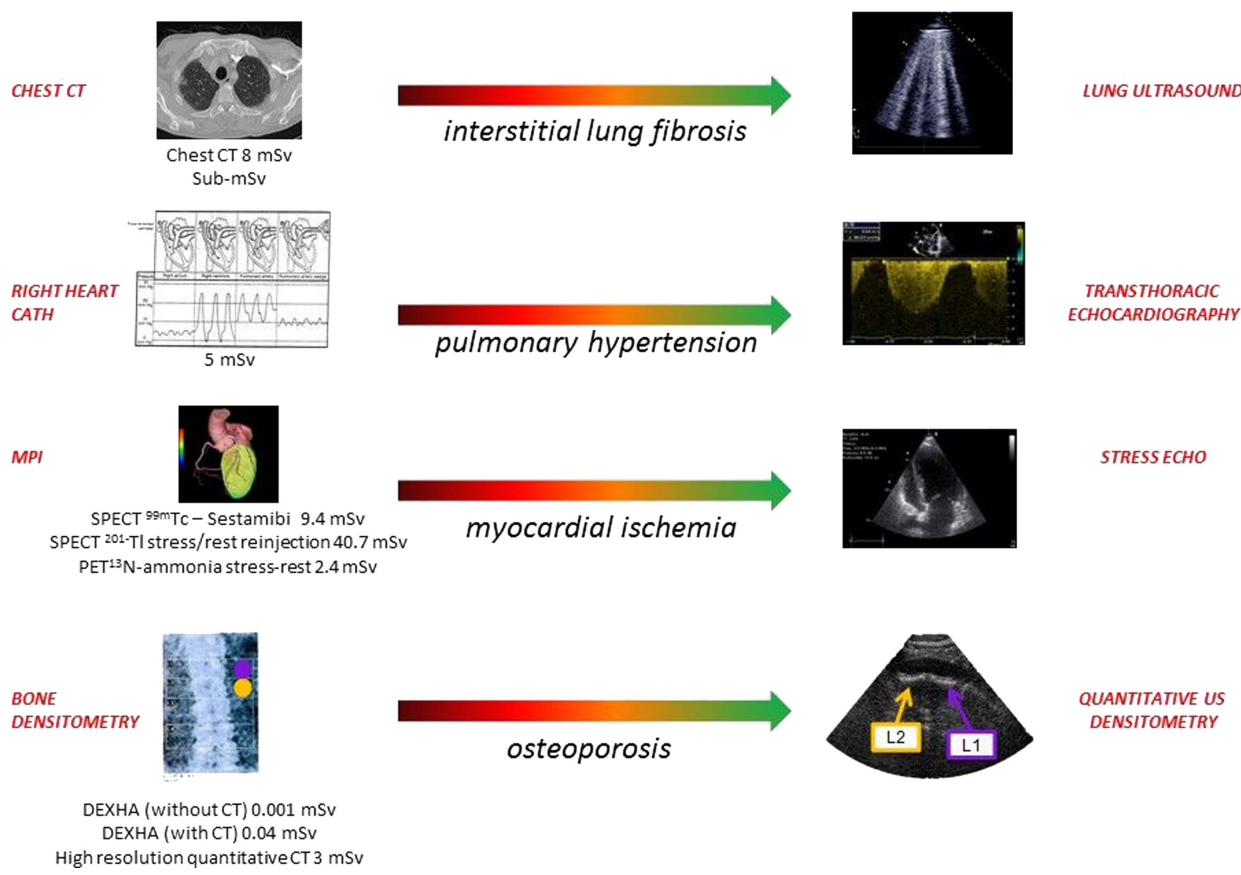


resting and exercise stress echocardiography can also rule out this pulmonary complication in $\mathrm{SSc}^{31}$ because patients with a normal stress echo are unlikely to develop pulmonary hypertension in the following years. ${ }^{32}$ Myocardial perfusion scintigraphy is still widely used for diagnosis and prognostic stratification in coronary artery disease, but its utilisation has fallen in recent years due to radiation exposure concerns $^{33}$ and the dissemination of alternative, equally accurate radiation-free techniques such as stress MRI and stress echocardiography. $^{34} 35$ Osteoporosis is usually assessed using radiological techniques, but recently quantitative ultrasound densitometric methods have become available. ${ }^{36}$ Lung fibrosis is ideally tracked with chest CT, but limited section CT can be substituted, and recent research suggests that ultrasound and MRI may be an accurate alternative ${ }^{37}$ even for early stages of disease, in particular in the follow-up and screening of SSc patients; with the additional benefits that it is a simple, low-cost and portable technique. ${ }^{38}$ When lack of available technology and expertise do not allow the use of an alternative radiationfree technique, great care should be taken to use the lowest dose radiation methods: for myocardial perfusion imaging, sestamibi is superior to thallium with singlephoton emission computed tomography (SPECT), and positron emission tomography (PET) (with rubidium or water) is better than SPECT; for lung fibrosis, low-dose and even sub-mSv CT are now available; for right heart catheterisation, optimised use of fluoroscopy can reduce radiation exposure fivefold; and for osteoporosis, high resolution peripheral quantitative CT-based methods are more accurate with substantially less radiation dose than dual X-ray absorptiometry technique (figure 2).

Because of the numerous sources of variability, there is no clear threshold between acceptable and unacceptable exposures for any given examination, but it is reasonable to postulate that any dose administered that has not been considered carefully with risk-benefit analysis and recorded in the patient's records is unacceptable. ${ }^{39}$ It is wise for the rheumatologist to be well aware of the typical radiation doses and suspected risks of medical radiation, and to prescribe medical imaging while taking into account the principles of justification and optimisation, considering that 'each patient should get the right imaging exam, at the right time, with the right radiation dose' in order to minimise the risk of iatrogenic cancer development. ${ }^{40}$ A prudent use of radiation is especially important in paediatric patients, who are particularly vulnerable to radiation damage. ${ }^{41}$

\section{Competing interests None.}

Provenance and peer review Commissioned; externally peer reviewed.

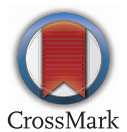

To cite Picano E, Semelka R, Ravenel J, et al. Ann Rheum Dis 2014;73:2065-2068.

Received 6 September 2014

Accepted 12 September 2014

Published Online First 9 October 2014

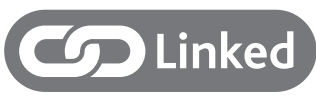

http://dx.doi.org/10.1136/annrheumdis-2014-205637

Ann Rheum Dis 2014;73:2065-2068.

doi:10.1136/annrheumdis-2014-206585

\section{REFERENCES}

1 Onishi A, Sugiyama D, Kumagai S, et al. Cancer incidence in systemic sclerosis: meta-analysis of population-based cohort studies. Arthritis Rheum 2013;65:1913-21.

2 Bonifazi M, Tramacere I, Pomponio G, et al. Systemic sclerosis (scleroderma) and cancer risk: systematic review and meta-analysis of observational studies. Rheumatology (Oxford) 2013;52:143-54.

3 Zhang JQ, Wan YN, Peng WJ, et al. The risk of cancer development in systemic sclerosis: $\mathrm{A}$ meta-analysis. Cancer Epidemiol 2013:37:523-7.

4 Bernatsky S, Ramsey-Goldman R, Labrecque J, et al. Cancer risk in systemic lupus: an updated international multi-centre cohort study. J Autoimmun 2013;42:130-5.

5 Mercer LK, Davies R, Galloway JB, et al. British Society for Rheumatology Biologics Register (BSRBR) Control Centre Consortium. Risk of cancer in patients receiving non-biologic disease-modifying therapy for rheumatoid arthritis compared with the UK general population. Rheumatology (Oxford) 2013;52:91-8.

6 Pouplard C, Brenaut E, Horreau C, et al. Risk of cancer in psoriasis: a systematic review and meta-analysis of epidemiological studies. J Eur Acad Dermatol Venereol 2013:27:36-46.

7 Turesson C, Matteson EL. Malignancy as a comorbidity in rheumatic diseases. Rheumatology (Oxford) 2013;52:5-14.

8 Frauenfelder T, Winklehner A, Nguyen TDN, et al. Screening for interstitial lung disease in systemic sclerosis: performance of high resolution computed tomography with limited number of slices - a prospective study. Ann Rheum Dis 2014;73: 2069-73.

9 Mettler FA Jr, Bhargavan M, Faulkner K, et al. Radiologic and nuclear medicine studies in the United States and worldwide: frequency, radiation dose, and comparison with other radiation sources1950-2007. Radiology 2009;253:520-31.

10 Mettler FA Jr, Huda W, Yoshizumi TT, et al. Effective doses in radiology and diagnostic nuclear medicine: a catalog. Radiology 2008:248:254-63.

11 Health Risks from Exposure to Low Levels of Ionizing Radiation. BEIR VII Phase 2. Committee to assess health risks from exposure to low levels of ionizing radiation. The National Academies Press, 2008. http://www.nap.edu
12 Picano E, Matucci-Cerinic M. Unnecessary radiation exposure from medical imaging in the rheumatology patient. Rheumatology (Oxford) 2011;50:1537-9.

13 Pearce MS, Salotti JA, Little MP, et al. Radiation exposure from CT scans in childhood and subsequent risk of leukaemia and brain tumours: a retrospective cohort study. Lancet 2012;380:499-505.

14 Mathews JD, Forsythe AV, Brady Z, et al. Cancer risk in 680,000 people exposed to computed tomography scans in childhood or adolescence: data linkage study of 11 million Australians. BMJ 2013;346: f2360.

15 Eisenberg MJ, Afilalo J, Lawler PR, et al. Cancer risk related to low-dose ionizing radiation from cardiac imaging in patients after acute myocardial infarction. CMAJ 2011:183:430-6.

16 ICRP Publication 103. The 2007 Recommendations of the International Commission on Radiological Protection. http://www.icrp.org/docs/lCRP Publication_103-Annals_of_the_ICRP_37(2-4)-Free_ extract.pdf

17 Avouac J, Huscher D, Furst DE, et al. for the EPOSS group. Expert consensus for performing right heart catheterisation for suspected pulmonary arterial hypertension in systemic sclerosis: a Delphi consensus study with cluster analysis. Ann Rheum Dis 2014:73:191-7.

18 Strollo D, Goldin J. Imaging lung disease in systemic sclerosis. Curr Rheumatol Rep 2010:12:156-61.

19 Baron M, Feiglin D, Hyland R, et al. 67-Gallium lung scans in progressive systemic sclerosis. Arthritis Rheum 1983;26:969-74.

20 Ishida R, Murata Y, Sawada Y, et al. Thallium-201 myocardial SPET in patients with collagen disease. Nucl Med Commun 2000:21:729-34.

21 Wessberg P, Danielson BI, Willén J. Comparison of Cobb angles in idiopathic scoliosis on standing radiographs and supine axially loaded MRI. Spine 2006:31:3039-44.

22 Pijpe A, Andrieu N, Easton DF, et al. GENEPSO; EMBRACE; HEBON. Exposure to diagnostic radiation and risk of breast cancer among carriers of BRCA1/2 mutations: retrospective cohort study (GENE-RAD-RISK). BMJ 2012;345:e5660.

23 McCurdy D, Tai LQ, Frias S, et al. Delayed repair of DNA damage by ionizing radiation in cells from patients with juvenile systemic lupus erythematosus and rheumatoid arthritis. Radiat Res 1997;147:48-54.

24 Abu-Shakra M, Lee P. Exaggerated fibrosis in patients with systemic sclerosis (scleroderma) following radiation therapy. J Rheumatol 1993;20:1601-3.

25 Yarnold J, Brotons MC. Pathogenetic mechanisms in radiation fibrosis. Radiother Oncol 2010;97:149-61.

26 Radis CD, Kahl LE, Baker GL, et al. Effects of cyclophosphamide on the development of malignancy and on long-term survival of patients with rheumatoid arthritis. A 20-year follow-up study. Arthritis Rheum 1995;38:1120-7.

27 Dobrzyńska MM. The effects in mice of combined treatments to $X$-rays and antineoplastic drugs in the Comet assay. Toxicology 2005;207:331-8.

28 ICRP Statement on Tissue Reactions / Early and Late Effects of Radiation in Normal Tissues and Organs-Threshold Doses for Tissue Reactions in a Radiation Protection Context. ICRP Publication 118 Ann. ICRP 41. 2012. http://www. icrp.org/publication.asp?id=ICRP\%20Publication\% 20118

29 Brunborg G, Holme JA, Hongslo JK. Inhibitory effects of paracetamol on DNA repair in mammalian cells. Mutat Res 1995;342:57-170.

30 Winklehner $A$, et al. Screening for interstitial lung disease in systemic sclerosis: the diagnostic accuracy of HRCT image series with high increment and reduced number of slices. Ann Rheum Dis 2012;71:549-52. 
31 Gargani L, Pignone A, Agoston G, et al.: Clinical and echocardiographic correlations of exercise-induced pulmonary hypertension in systemic sclerosis: a multicenter study. Am Heart J 2013;165:200-7.

32 Picano E, Pellikka PA. Stress echo applications beyond coronary artery disease. Eur Heart J 2014;35:1033-40.

33 Smith-Bindman R, Miglioretti DL, Johnson E, et al. Use of diagnostic imaging studies and associated radiation exposure for patients enrolled in large integrated health care systems, 1996-2010. JAMA 2012;307:2400-9.

34 Picano E. Stress echocardiography: a historical perspective. Am J Med 2003:114:126-30.

35 Sicari R, Nihoyannopoulos P, Evangelista A, et al. European Association of Echocardiography. Stress
Echocardiography Expert Consensus StatementExecutive Summary: European Association of Echocardiography (EAE) (a registered branch of the ESC). Eur Heart J 2009;30:278-89.

36 Link TM. Osteoporosis imaging: state of the art and advanced imaging. Radiology 2012;263:3-17.

37 Gargani L, Doveri M, D'Errico L, et al. Ultrasound lung comets in systemic sclerosis: a chest sonography hallmark of pulmonary interstitial fibrosis.

Rheumatology (Oxford) 2009;48:1382-7.

38 Barskova T, Gargani L, Guiducci S, et al. Lung ultrasound for the screening of interstitial lung disease in very early systemic sclerosis. Ann Rheum Dis 2013:72:390-5.

39 Picano E, Vañó E, Rehani MM, et al. The appropriate and justified use of medical radiation in cardiovascular imaging. A position document of the ESC associations of cardiovascular imaging, percutaneous cardiovascular interventions and electrophysiology. Eur Heart $J$ 2014;35:665-72.

40 U.S. Food and Drug Administration. Initiative to Reduce Unnecessary Radiation Exposure from Medical Imaging. February 2010. http://www.fda. gov/downloads/Radiation-EmittingProducts/ RadiationSafety/RadiationDoseReduction/ UCM200087.pdf

41 Andreassi MG, Picano E. Reduction of radiation to children. Our responsibility to change. Editorial comment. Circulation 2014:130:135-7. 